\title{
Randomized phase II trial of nimotuzumab plus irinotecan versus irinotecan alone as second-line therapy for patients with advanced gastric cancer
}

\author{
Taroh Satoh • Kyung Hee Lee - Sun Young Rha Yasutsuna Sasaki - Se Hoon Park • Yoshito Komatsu • \\ Hirofumi Yasui - Tae-You Kim $\cdot$ Kensei Yamaguchi $\cdot$ Nozomu Fuse $\cdot$ Yasuhide Yamada Takashi Ura \\ Si-Young Kim • Masaki Munakata $\cdot$ Soh Saitoh $\cdot$ Kazuto Nishio Satoshi Morita Eriko Yamamoto • \\ Qingwei Zhang · Jung-mi Kim • Yeul Hong Kim • Yuh Sakata
}

Received: 14 March 2014/ Accepted: 28 July 2014/Published online: 5 September 2014

(C) The Author(s) 2014. This article is published with open access at Springerlink.com

\begin{abstract}
Background This multicenter, randomized phase II trial was conducted to compare the efficacy and safety of nimotuzumab plus irinotecan (N-IRI) versus irinotecan alone (IRI) in patients with advanced gastric cancer (AGC) showing disease progression after previous 5-fluorouracilbased therapy.

Methods Irinotecan-naive patients $(n=82)$ received $\mathrm{N}$-IRI (nimotuzumab $400 \mathrm{mg}$ weekly plus irinotecan

Presented in part at the 2011 American Society of Clinical Oncology Gastrointestinal Cancers Symposium, January 20-22, 2011, San Francisco, California, the Ninth International Gastric Cancer Congress, April 20-23, 2011, Seoul, Republic of Korea, and the Fourth West Japan Oncology Group International Symposium, April 6-7, 2012, Fukuoka, Japan.
\end{abstract}

Trial registration ID: JapicCTI-090849.

\section{T. Satoh}

Medical Oncology, Kinki University Faculty of Medicine,

Osaka, Japan

\section{K. H. Lee}

Hemato-Oncology, Internal Medicine, Yeungnam University

Hospital, Daegu, Republic of Korea

\section{S. Y. Rha}

Medical Oncology, Internal Medicine, Yonsei Cancer Center,

Yonsei Cancer Research Institute, Yonsei University College of

Medicine, Seoul, Republic of Korea

Y. Sasaki

Medical Oncology, Saitama Medical University International

Medical Center, Saitama, Japan

S. H. Park

Division of Hematology-Oncology, Department of Medicine, Sungkyunkwan University Samsung Medical Center, Seoul,

Republic of Korea
$150 \mathrm{mg} / \mathrm{m}^{2}$ biweekly) or IRI (irinotecan $150 \mathrm{mg} / \mathrm{m}^{2}$ biweekly) until disease progression. The primary endpoint was progression-free survival (PFS), and the secondary endpoints were overall survival (OS), response rate (RR), safety, tolerability, and the correlation between efficacy and tumor epidermal growth factor receptor (EGFR) expression.

Results Of 83 patients, 40 and 43 patients were randomly assigned to the N-IRI and IRI groups, respectively. In the N-IRI/IRI treatment group, median PFS was 73.0/ 85.0 days $(P=0.5668)$, and median OS and RR at 18 months were $250.5 / 232.0$ days $(P=0.9778)$ and $18.4 /$ $10.3 \%$, respectively. Median PFS and OS in the EGFR $2+/ 3+$ subgroups were $118.5 / 59.0$ and $358.5 / 229.5$ days, respectively. The RR was $33.3 / 0.0 \%$ in the N-IRI/IRI treatment group. The incidence of grade 3 or higher adverse events was $77.5 / 64.3 \%$. No adverse events of

\section{Y. Komatsu}

Division of Cancer Chemotherapy, Hokkaido University

Hospital, Hokkaido, Japan

H. Yasui

Division of GI Oncology, Shizuoka Cancer Center, Shizuoka, Japan

\section{T.-Y. Kim}

Hematology-Oncology, Internal Medicine, Seoul National

University Hospital, Seoul, Republic of Korea

K. Yamaguchi

Department of Gastroenterology, Saitama Cancer Center,

Saitama, Japan

N. Fuse

Gastrointestinal Oncology, National Cancer Center Hospital East, Chiba, Japan 
grade 3 or higher skin rash or grade 3 or higher infusionrelated reaction were reported.

Conclusions There was no superiority of N-IRI over IRI alone in terms of PFS in 5-fluorouracil-refractory AGC patients. However, N-IRI showed potential improvement in the EGFR $2+/ 3+$ subgroup based on improved RR, PFS, and OS.

Keywords Nimotuzumab · Anti-EGFR · Irinotecan · Second-line therapy $\cdot$ Advanced gastric cancer

\section{Introduction}

Patients with unresectable gastric cancer receiving the best supportive care have poor outcomes, with median survival times ranging from 3 to 5 months [1, 2]. In the metastatic disease setting, palliative chemotherapy improves survival compared with supportive care alone, with combined drug therapy yielding the best results [1-3]. Although there is no universally accepted standard treatment for advanced gastric cancer (AGC), several combination regimens have been used as first-line treatment, including epirubicin-oxaliplatin-capecitabine [4], cisplatin-capecitabine [5], cisplatin-S-1 [6], cisplatin-5-fluorouracil, and docetaxelcisplatin-5-fluorouracil [7]. However, the median survival has not exceeded 8-13 months [1-7], and second-line treatments need to be established. Irinotecan $[8,9]$ or paclitaxel monotherapy is commonly used for AGC patients as second-line treatment, especially in Japan and Korea. Because of the limitations of the current therapies, addition of molecular-targeted drugs, particularly to chemotherapies with acceptable toxicities, may improve

\footnotetext{
Y. Yamada

Gastrointestinal Oncology, National Cancer Center Hospital,

Tokyo, Japan

T. Ura

Department of Clinical Oncology, Aichi Cancer Center Hospital,

Aichi, Japan

S.-Y. Kim

Department of Medical Oncology and Hematology, Kyung Hee University Hospital, Seoul, Republic of Korea

M. Munakata · Y. Sakata

Internal Medicine, Misawa Municipal Hospital, Aomori, Japan

S. Saitoh

Medical Oncology and Gastroenterology, Aomori Prefectural

Central Hospital, Aomori, Japan

K. Nishio

Department of Genome Biology, Kinki University School of

Medicine, Osaka, Japan
}

the outcomes. The ToGA trial demonstrated that the addition of trastuzumab to standard chemotherapy in patients with human EGFR-2 (HER-2)-overexpressing tumors improved overall survival (OS) and progressionfree survival (PFS) [10].

Epidermal growth factor receptor (EGFR) is known to be expressed in a variety of tumors [11]. Approximately $30 \%$ of gastric cancers are reported to show EGFR overexpression [12, 13]. EGFR signaling pathways are frequently dysregulated in gastric cancer, thereby serving as attractive therapeutic targets.

Nimotuzumab, a recombinant humanized monoclonal immunoglobulin $\mathrm{G}_{1}$ antibody against human EGFR (HER1), blocks the binding of epidermal growth factor (EGF) and transforming growth factor- $\alpha$ to EGFR. This mechanism regulates antibody-dependent cellular cytotoxicity and complement-dependent cytotoxicity, inhibiting tumor cell growth and angiogenesis and inducing apoptosis [14-17]. In a previous phase I study in Japan, the safety and tolerability of nimotuzumab were investigated up to $400 \mathrm{mg}$ doses weekly [18]. When combined with radiotherapy or chemoradiotherapy, nimotuzumab exerts clinical efficacy against head and neck cancers, gliomas, and non-small cell lung cancer (NSCLC) $[17,19,20]$. Additionally, because of the low frequency of severe dermatological toxicity, nimotuzumab is expected to improve the quality of life.

The present study was an open-label, phase II collaborative study between Japan and Korea. The primary objective was to compare PFS following combined nimotuzumab plus irinotecan therapy (N-IRI) and irinotecan monotherapy (IRI) in patients with unresectable or recurrent gastric cancer refractory to 5-fluorouracil-based therapy.

\footnotetext{
S. Morita

Department of Biostatics and Epidemiology, Yokohama City University, Kanagawa, Japan

E. Yamamoto

Clinical Development Department II, Daiichi Sankyo Co., Ltd., Tokyo, Japan

Q. Zhang

Clinical Data and Biostatistics Department, Daiichi Sankyo Co., Ltd., Tokyo, Japan

J. Kim

Medical and Regulatory Affairs Department, Kuhnil Pharm. Co., Ltd., Seoul, Republic of Korea

Y. H. Kim (ه)

Department of Internal Medicine, Section of Hemato-Oncology, Korea University Anam Hospital, 126-1 Anam-dong 5ga, Seongbuk-gu, Seoul 136-705, Republic of Korea e-mail: yhk0215@korea.ac.kr
} 


\section{Materials and methods}

\section{Patients}

Patients in Japan and Korea were enrolled in this multicenter, open-label, randomized phase II trial. Patients with histologically confirmed AGC refractory to previous 5-fluorouracil-based chemotherapy for metastatic disease were eligible. Other major inclusion criteria were as follows: age of 20-75 years; adequate organ function; and Eastern Cooperative Oncology Group performance status (ECOG PS) 0 or 1. Major exclusion criteria were prior exposure to irinotecan or EGFR-directed therapy, and significant comorbidities, such as diarrhea, interstitial pneumonia, or pulmonary fibrosis.

The trial was conducted in accordance with the Declaration of Helsinki. All the patients provided written informed consent. The institutional review boards or ethics committees of all participating centers reviewed and approved the protocol.

\section{Study treatment}

Patients were randomly assigned at a ratio of $1: 1$ to the $\mathrm{N}$-IRI or IRI group by a computer program on the basis of the resection status of the primary tumor (inoperable advanced/postoperative recurrent) and study site, using the random permuted blocks method. Neither the patients nor the investigators were blinded to the treatment assignment.

Nimotuzumab (400 $\mathrm{mg}$ ) diluted in normal saline to a total volume of $250 \mathrm{ml}$ was administered once weekly by intravenous infusion over $30 \mathrm{~min}$. Irinotecan $\left(150 \mathrm{mg} / \mathrm{m}^{2}\right)$ was administered every 2 weeks. Treatment was continued until disease progression, appearance of unacceptable toxicity, or withdrawal of consent.

\section{Efficacy and safety assessments}

The primary endpoint was PFS following N-IRI versus IRI treatment. PFS was defined as the time from randomization to the day of documentation of progression or death, whichever was earlier. The secondary endpoints were OS, response rate (RR), disease control rate (DCR), safety, and tolerability. Tumor assessment by computed tomography was performed at baseline, and then every 4 weeks for the first 16 weeks, and every 6 weeks thereafter. Evaluation of tumors was performed by an independent Efficacy Evaluation Committee using RECIST 1.0. Adverse events were assessed according to the National Cancer Institute's Common Terminology Criteria for Adverse Events, version 3.0 .
Exploratory biomarker analysis

EGFR protein expression levels, EGFR gene amplification status, $K$-ras mutations, and HER-2 protein expression levels were measured in tissue specimens from tumors obtained from patients who had provided informed consent for exploratory biomarker analysis. The tumor tissues were centrally tested and classified. EGFR expression was analyzed using an immunohistochemistry (IHC) staining kit (EGFR PharmDX; Dako, Copenhagen, Denmark) and classified into four categories $(0,1+, 2+$, and $3+)$, as previously described [21]. The EGFR gene copy number was measured by fluorescence in situ hybridization (FISH), as reported previously [22]. For K-ras mutation analysis, DNA was extracted from formalin-fixed paraffin-embedded tumor samples. The sequences of $K$-ras codons 12 and 13 and the surrounding region of the gene were analyzed by conventional polymerase chain reaction followed by direct sequencing. The expression status of HER-2 was analyzed using the HercepTest kit (Dako) and classified into four categories $(0,1+, 2+$, and $3+)$.

Statistical analysis

The reported median PFS in AGC patients treated with irinotecan or paclitaxel as second-line chemotherapy is 2.1-2.6 months [23-25]. For an exploratory study, if the median PFS times for N-IRI and IRI therapy are assumed to be 4.5 and 2.5 months, respectively, then 32 patients per treatment arm would be required to detect a difference with $80 \%$ power at a $10 \%$ significance level using a one-sided log-rank test of the equality of survival curves. Assuming a dropout rate of $20 \%$, the number of patients per treatment group was set at 40, with a total sample size of at least 80 patients. The median PFS was calculated with the $95 \%$ confidence interval (CI) for both treatment groups. Logrank tests were performed to evaluate differences in PFS with the significance level set at $10 \%$ (one sided). Primary statistical analysis of the efficacy endpoint was performed 6 months after registration of the last patient for the study.

For subgroup analyses of PFS and OS, the hazard ratio (HR) and $95 \%$ CI within each subgroup were displayed in forest plots. In both treatment groups, the Kaplan-Meier method was used to plot the survival curves and estimate the cumulative incidence from the day of registration to death, as well as the cumulative incidence to disease progression. For evaluation of efficacy, point estimates were calculated for the RR and DCR and compared using the chi-squared test. Efficacy endpoints were analyzed using the full analysis set, safety endpoints were analyzed using the safety analysis set, and pharmacokinetic analyses were performed using the pharmacokinetic analysis set. 


\section{Results}

\section{Patients}

A total of 83 patients were randomized from September 2008 to December 2009. Of these patients, 82 were included in the safety and efficacy analysis population (1 patient from the IRI group did not have a target lesion and did not receive irinotecan; Fig. 1). At the 18-month followup after registration of the last patient for the study, the median nimotuzumab and irinotecan exposure in the N-IRI group was 71.5 days (range, 8-947) and 60.5 days (range, 1-268), respectively, and the median irinotecan exposure in the IRI group was 57.0 days (range, 1-953). The median follow-up period was 242.5 days (range, 22-955). Patient demographics, including the UGT1A1 subtype, were well matched between the two treatment groups (Table 1).

Of the 83 patients, 48 patients had provided informed consent for exploratory biomarker analysis and submitted tumor samples. The EGFR protein expression level was detected in the assessable tumor tissues of 47 patients (57.3\% of the full analysis set population) (Table 2).

\section{Efficacy}

A total of 77 patients $(n=38$ in the N-IRI group and $n=39$ in the IRI group) were evaluable for radiologic tumor responses by an Independent Efficacy Evaluation Committee. PFS evaluated at 6 months after registration of the last patient was not significantly different between the treatment groups [median (95\% CI), 73.0 (55.0-112.0) days in the N-IRI group vs. 85.0 (37.0-93.0) days in the IRI group; HR (95\% CI), 0.860 (0.516-1.435), $P=0.5668$ ] (Fig. 2).

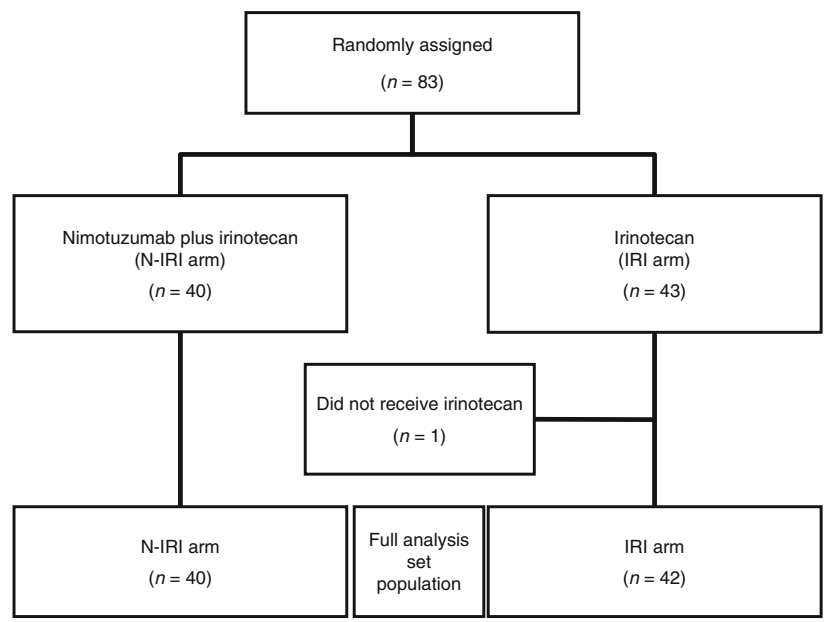

Fig. 1 CONSORT diagram. $N$-IRI nimotuzumab plus irinotecan, IRI irinotecan alone
By 18 months after registration of the last patient, 34 patients from each group had died and the 18-month OS was not significantly different between the treatment groups [median (95\% CI), 250.5 (171.0-306.0) days in the N-IRI group vs. 232.0 (148.0-319.0) days in the IRI group; HR (95 \% CI), 0.994 (0.618-1.599), $P=0.9778]$. There

Table 1 Baseline characteristics of the patients

\begin{tabular}{|c|c|c|c|c|c|c|}
\hline \multirow[t]{2}{*}{ Characteristics } & \multicolumn{2}{|c|}{$\begin{array}{l}\text { N-IRI } \\
\text { arm } \\
(n=40)\end{array}$} & \multicolumn{2}{|c|}{$\begin{array}{l}\text { IRI arm } \\
(n=42)\end{array}$} & \multicolumn{2}{|c|}{$\begin{array}{l}\text { Total } \\
(n=82)\end{array}$} \\
\hline & $n$ & $\%$ & $n$ & $\%$ & $n$ & $\%$ \\
\hline \multicolumn{7}{|l|}{ Age (years) } \\
\hline Median & \multicolumn{2}{|c|}{60.0} & \multicolumn{2}{|c|}{63.5} & \multicolumn{2}{|c|}{61.5} \\
\hline Range & \multicolumn{2}{|c|}{$27-75$} & \multicolumn{2}{|c|}{$32-75$} & \multicolumn{2}{|c|}{$27-75$} \\
\hline \multicolumn{7}{|l|}{ Sex } \\
\hline Male & 33 & 82.5 & 33 & 78.6 & 66 & 80.5 \\
\hline Female & 7 & 17.5 & 9 & 21.4 & 16 & 19.5 \\
\hline \multicolumn{7}{|l|}{ ECOG performance status } \\
\hline 0 & 19 & 47.5 & 17 & 40.5 & 36 & 43.9 \\
\hline 1 & 21 & 52.5 & 25 & 59.5 & 46 & 56.1 \\
\hline \multicolumn{7}{|l|}{ Body weight (kg) } \\
\hline Median & \multicolumn{2}{|c|}{56.3} & \multicolumn{2}{|c|}{54.2} & \multicolumn{2}{|c|}{56.0} \\
\hline Range & \multicolumn{2}{|c|}{$42.0-81.4$} & \multicolumn{2}{|c|}{$37.5-107.0$} & \multicolumn{2}{|c|}{$37.5-107.0$} \\
\hline \multicolumn{7}{|c|}{ Resection status of the primary tumor } \\
\hline Inoperable advanced & 22 & 55.0 & 23 & 54.8 & 45 & 54.9 \\
\hline Postoperative recurrent & 18 & 45.0 & 19 & 45.2 & 37 & 45.1 \\
\hline \multicolumn{7}{|l|}{ Histological diagnosis, $n$} \\
\hline $\begin{array}{l}\text { Well/moderately } \\
\text { differentiated } \\
\text { adenocarcinoma }\end{array}$ & 15 & 37.5 & 19 & 45.2 & 34 & 41.5 \\
\hline $\begin{array}{l}\text { Poorly differentiated } \\
\text { adenocarcinoma }\end{array}$ & 21 & 52.5 & 17 & 40.5 & 38 & 46.3 \\
\hline Others & 4 & 10.0 & 6 & 14.3 & 10 & 12.2 \\
\hline \multicolumn{7}{|l|}{ Primary tumor site } \\
\hline Absent & 18 & 45.0 & 16 & 38.1 & 34 & 41.5 \\
\hline Present & 22 & 55.0 & 26 & 61.9 & 48 & 58.5 \\
\hline Gastroesophageal junction & 4 & 18.2 & 1 & 3.8 & 5 & 10.4 \\
\hline Gastric region & 18 & 81.8 & 25 & 96.2 & 43 & 89.6 \\
\hline \multicolumn{7}{|l|}{ Metastatic focus site } \\
\hline No & 1 & 2.5 & 0 & 0.0 & 1 & 1.2 \\
\hline Yes & 39 & 97.5 & 42 & 100.0 & 81 & 98.8 \\
\hline Lymph node & 25 & 64.1 & 25 & 59.5 & 50 & 61.7 \\
\hline Liver & 13 & 33.3 & 19 & 45.2 & 32 & 39.5 \\
\hline Lung & 3 & 7.7 & 6 & 14.3 & 9 & 11.1 \\
\hline Other & 19 & 48.7 & 18 & 42.9 & 37 & 45.7 \\
\hline \multicolumn{7}{|l|}{ UGT1A1 gene polymorphism } \\
\hline$* 1 / * 1, * 1 / * 6, * 1 / * 28$ & 38 & 95.0 & 39 & 92.9 & 77 & 93.9 \\
\hline$* 6 / * 6, * 28 / * 28, * 6 / * 28$ & 2 & 5.0 & 3 & 7.1 & 5 & 6.1 \\
\hline
\end{tabular}

$N$-IRI nimotuzumab plus irinotecan, IRI irinotecan alone, $n$ number of patients, ECOG Eastern Cooperative Oncology Group 
Table 2 EGFR and HER-2 protein expression levels identified by immunohistochemistry

\begin{tabular}{|c|c|c|c|c|c|c|c|c|}
\hline & \multicolumn{8}{|c|}{ EGFR } \\
\hline & \multicolumn{2}{|l|}{0} & \multicolumn{2}{|l|}{$1+$} & \multicolumn{2}{|c|}{$2+, 3+$} & \multicolumn{2}{|c|}{ Total } \\
\hline & $n$ & $\%$ & $n$ & $\%$ & $n$ & $\%$ & $n$ & $\%$ \\
\hline \multicolumn{9}{|l|}{ HER2 } \\
\hline 0 & 15 & 31.3 & 8 & 16.7 & 8 & 16.7 & 31 & 64.6 \\
\hline $1+$ & 2 & 4.2 & 2 & 4.2 & 5 & 10.4 & 9 & 18.8 \\
\hline $2+, 3+$ & 4 & 8.3 & 2 & 4.2 & 1 & 2.1 & 7 & 14.6 \\
\hline Total & 21 & 43.8 & 12 & 25.0 & 14 & 29.2 & $47^{\mathrm{a}}$ & 97.9 \\
\hline
\end{tabular}

EGFR epidermal growth factor receptor, $n$ number of patients, $H E R 2$ human EGFR-2

"One sample was "not detected"
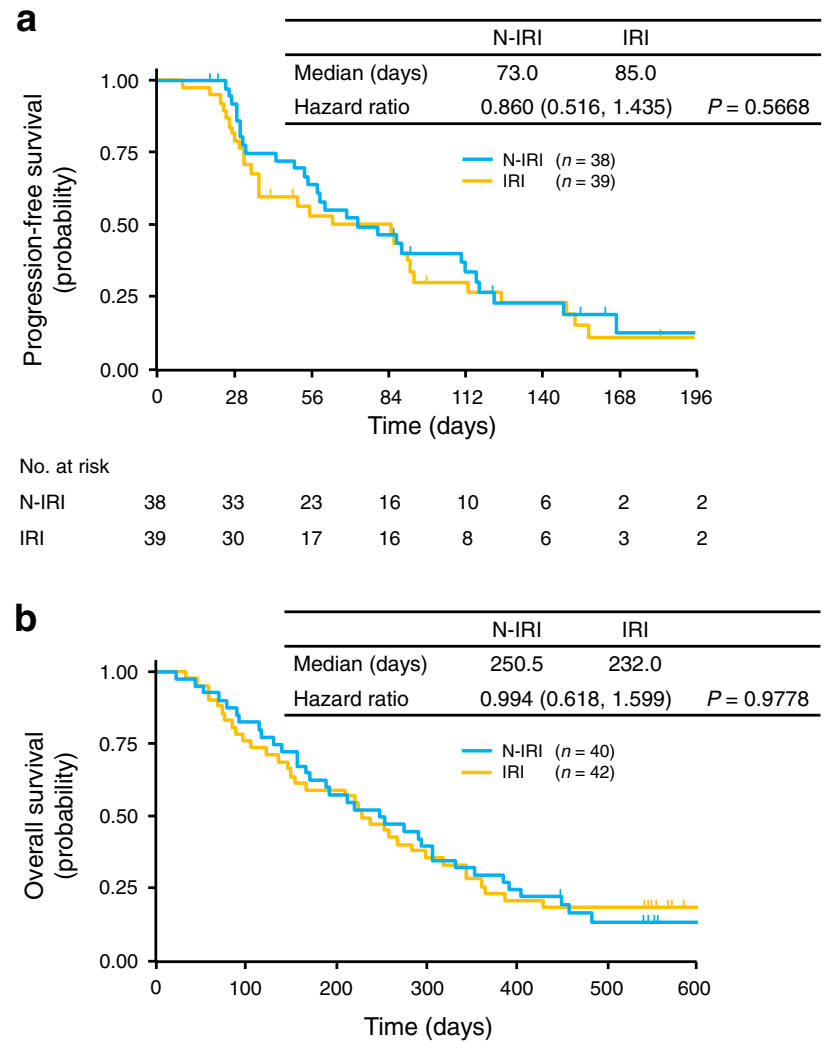

$\begin{array}{llllllll}\text { No. at risk } & & & & & & & \\ \text { N-IRI } & 40 & 33 & 23 & 16 & 10 & 5 & 1 \\ \text { IRI } & 42 & 32 & 25 & 15 & 9 & 8 & 1\end{array}$

Fig. 2 Kaplan-Meier estimates of progression-free survival (a) and overall survival (b). $N$-IRI nimotuzumab plus irinotecan, IRI irinotecan alone

was no significant difference in RR or DCR at the 18-month follow-up between the treatment groups (RR, $18.4 \%$ in the N-IRI group vs. $10.3 \%$ in the IRI group, $P=0.3060 ;$ DCR, $47.4 \%$ in the N-IRI group vs. $46.2 \%$ in the IRI group, $P=0.9150$ ).
PFS and OS in the various subgroups analyzed were not significantly different between the treatment groups (Fig. 3). However, the HR (IRI/N-IRI) in the EGFR 2+/3+ subgroup was lower than that in the entire treatment group. First, the median PFS (95\% CI) was 118.5 (87.0-not estimated) days for six patients in the N-IRI group vs. 59.0 (24.0-113.0) days for six patients in the IRI group [HR (95\% CI), 0.341 (0.080-1.457), $P=0.1293$ ] (Fig. 3). Second, the median OS (95\% CI) was 358.5 (274.0-458.0) days for six patients in the N-IRI group vs. 229.5 (58.0-387.0) days for eight patients in the IRI group [HR (95\% CI), 0.369 (0.110-1.242), $P=0.0944$ ] (Fig. 3). Furthermore, at the 18-month follow-up, the RR in the EGFR $2+/ 3+$ subgroup was $33.3 \%$ for six patients in the $\mathrm{N}$-IRI group vs. $0.0 \%$ for six patients in the IRI group, and the DCR in the corresponding groups was $83.3 \%$ and $33.3 \%$, respectively.

\section{Pharmacokinetics}

Pharmacokinetic analysis was conducted using data collected from 11 patients $(n=6$ from the N-IRI group and $n=5$ from the IRI group). The pharmacokinetic parameters of nimotuzumab were similar to those reported from a previous phase I study of nimotuzumab in Japanese patients with solid tumors [18].

\section{Safety}

Adverse events were reported in all the patients. Table 3 shows the incidence, by treatment group, of major adverse events occurring at a frequency of $\geq 15 \%$ in at least one group. The most common adverse events $(\geq 50 \%$ in at least one group) were neutropenia, diarrhea, nausea, alopecia, decreased appetite, fatigue, and leukopenia. A rash occurred in $25.0 \%(10 / 40)$ and $4.8 \%(2 / 42)$ of patients in the N-IRI and IRI groups, respectively. There were no cases with severe ( $\geq$ grade 3 ) skin toxicity, including severe rash. Grade 3 or higher adverse events were encountered in $77.5 \%$ of patients in the N-IRI group and $64.3 \%$ of patients in the IRI group. The most common grade 3 or higher adverse events $(\geq 10 \%$ in at least one group) were neutropenia, nausea, leukopenia, anemia, pneumonia, and decreased hemoglobin. The two pneumonia-related deaths in the N-IRI group were considered to be causally related to the study drug. All patients with pneumonia were evaluated by an Independent Data Monitoring Committee to detect pneumonitis. However, no cases of pneumonitis were identified.

The incidence of adverse events resulting in discontinuation of irinotecan was $15.0 \%(6 / 40)$ in the N-IRI group and $16.7 \%(7 / 42)$ in the IRI group, with no significant difference between the two groups. The 


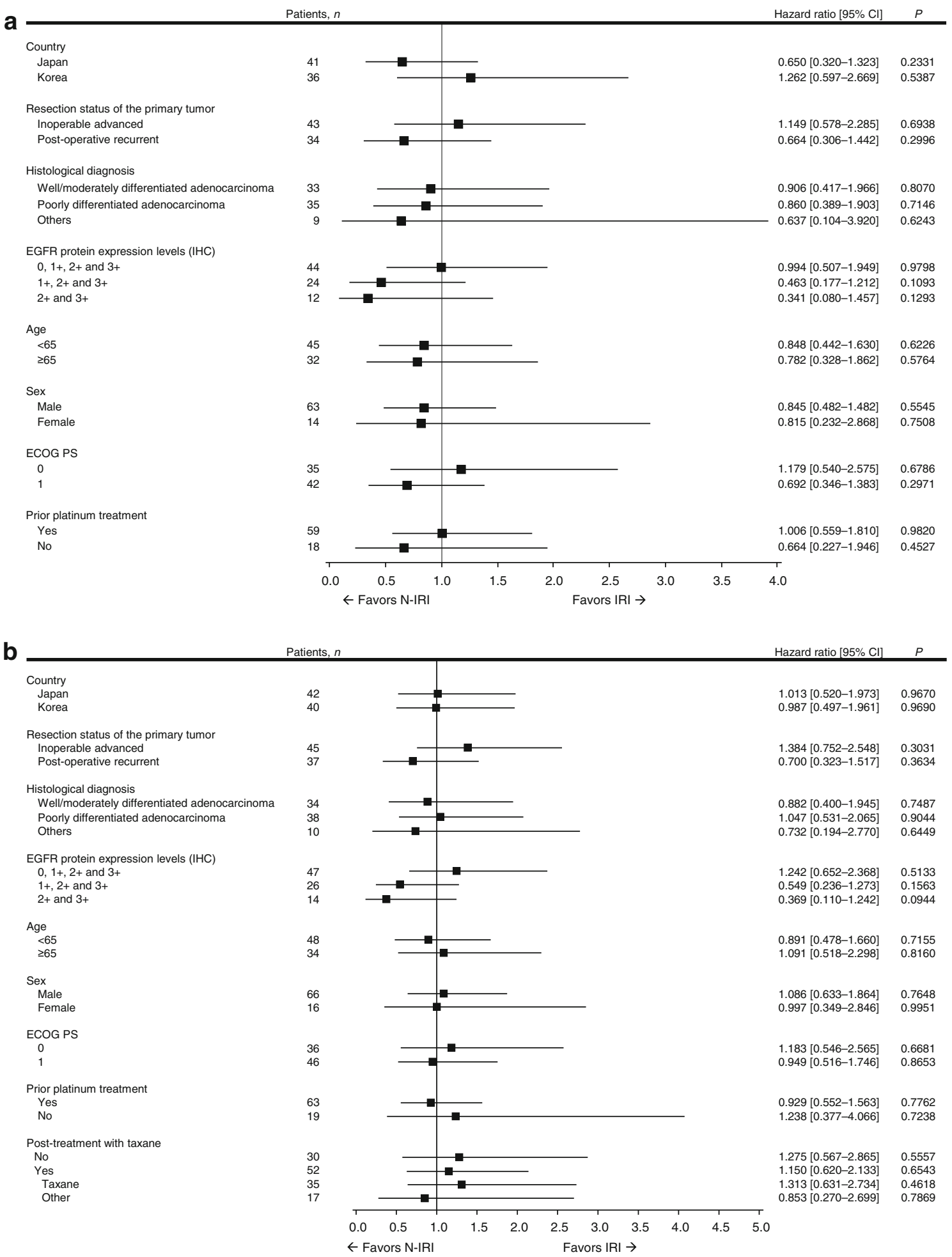

Fig. 3 Subset forest plots for progression-free survival (a) and overall survival (b). N-IRI nimotuzumab plus irinotecan, IRI irinotecan alone, EGFR epidermal growth factor receptor, IHC immunohistochemistry, ECOG Eastern Cooperative Oncology Group, PS performance status

incidence of adverse events resulting in discontinuation of nimotuzumab was $7.5 \%(3 / 40)$ in the N-IRI treatment group.
Adverse events were reported for all patients in the EGFR $2+/ 3+$ subgroup, and no significant difference was found in the frequency of adverse events between the IRI 
Table 3 Adverse events occurring at an incidence of $\geq 15 \%$ in each treatment arm

\begin{tabular}{|c|c|c|c|c|c|c|c|c|}
\hline \multirow[t]{3}{*}{ Adverse event } & \multicolumn{4}{|c|}{ N-IRI $(n=40)$} & \multicolumn{4}{|c|}{ IRI $(n=42)$} \\
\hline & \multicolumn{2}{|c|}{$\begin{array}{l}\text { All } \\
\text { grades }\end{array}$} & \multicolumn{2}{|c|}{$\begin{array}{l}\geq \text { Grade } \\
3\end{array}$} & \multicolumn{2}{|c|}{$\begin{array}{l}\text { All } \\
\text { grades }\end{array}$} & \multicolumn{2}{|c|}{$\begin{array}{l}\geq \text { Grade } \\
3\end{array}$} \\
\hline & $n$ & $\%$ & $n$ & $\%$ & $n$ & $\%$ & $n$ & $\%$ \\
\hline \multicolumn{9}{|c|}{ Infections and infestations } \\
\hline Pneumonia & 8 & 20.0 & 4 & 10.0 & 1 & 2.4 & 0 & 0.0 \\
\hline \multicolumn{9}{|c|}{ Blood and lymphatic system disorders } \\
\hline Anemia & 7 & 17.5 & 5 & 12.5 & 4 & 9.5 & 3 & 7.1 \\
\hline Leukopenia & 20 & 50.0 & 6 & 15.0 & 15 & 35.7 & 4 & 9.5 \\
\hline Lymphopenia & 7 & 17.5 & 3 & 7.5 & 4 & 9.5 & 0 & 0.0 \\
\hline Neutropenia & 29 & 72.5 & 18 & 45.0 & 23 & 54.8 & 16 & 38.1 \\
\hline Thrombocytopenia & 1 & 2.5 & 0 & 0.0 & 7 & 16.7 & 3 & 7.1 \\
\hline \multicolumn{9}{|c|}{ Metabolism and nutrition disorders } \\
\hline Hypoalbuminemia & 7 & 17.5 & 0 & 0.0 & 5 & 11.9 & 1 & 2.4 \\
\hline Decreased appetite & 22 & 55.0 & 3 & 7.5 & 26 & 61.9 & 3 & 7.1 \\
\hline \multicolumn{9}{|c|}{ Gastrointestinal disorders } \\
\hline Abdominal pain & 14 & 35.0 & 0 & 0.0 & 14 & 33.3 & 3 & 7.1 \\
\hline Constipation & 12 & 30.0 & 0 & 0.0 & 12 & 28.6 & 0 & 0.0 \\
\hline Diarrhea & 25 & 62.5 & 3 & 7.5 & 25 & 59.5 & 2 & 4.8 \\
\hline Nausea & 25 & 62.5 & 6 & 15.0 & 25 & 59.5 & 4 & 9.5 \\
\hline Stomatitis & 6 & 15.0 & 0 & 0.0 & 5 & 11.9 & 0 & 0.0 \\
\hline Vomiting & 17 & 42.5 & 3 & 7.5 & 13 & 31.0 & 2 & 4.8 \\
\hline \multicolumn{9}{|c|}{ Skin and subcutaneous tissue disorders } \\
\hline Alopecia & 23 & 57.5 & 0 & 0.0 & 15 & 35.7 & 0 & 0.0 \\
\hline Rash & 10 & 25.0 & 0 & 0.0 & 2 & 4.8 & 0 & 0.0 \\
\hline \multicolumn{9}{|c|}{ General disorders and administration site conditions } \\
\hline Asthenia & 7 & 17.5 & 1 & 2.5 & 9 & 21.4 & 1 & 2.4 \\
\hline Fatigue & 21 & 52.5 & 3 & 7.5 & 15 & 35.7 & 3 & 7.1 \\
\hline Pyrexia & 8 & 20.0 & 0 & 0.0 & 13 & 31.0 & 0 & 0.0 \\
\hline \multicolumn{9}{|l|}{ Investigations } \\
\hline $\begin{array}{l}\text { Alanine } \\
\text { aminotransferase } \\
\text { increased }\end{array}$ & 8 & 20.0 & 1 & 2.5 & 6 & 14.3 & 1 & 2.4 \\
\hline $\begin{array}{l}\text { Aspartate } \\
\text { aminotransferase } \\
\text { increased }\end{array}$ & 7 & 17.5 & 1 & 2.5 & 7 & 16.7 & 1 & 2.4 \\
\hline $\begin{array}{c}\text { Hemoglobin } \\
\text { decreased }\end{array}$ & 11 & 27.5 & 4 & 10.0 & 13 & 31.0 & 6 & 14 \\
\hline Weight decreased & 12 & 30.0 & 2 & 5.0 & 8 & 19.0 & 0 & 0.0 \\
\hline
\end{tabular}

Number of patients, incidence of adverse events, and incidence of grades 3-5 adverse events

$N$-IRI nimotuzumab plus irinotecan, IRI irinotecan alone, $n$ number of patients

and N-IRI groups in the EGFR $2+/ 3+$ subgroup analysis. The incidence of adverse events in the EGFR $2+13+$ subgroup was similar to that in all randomized patients. In the EGFR $2+/ 3+$ subgroup, rash of grade 1 or 2 occurred in $50.0 \%(3 / 6)$ and $0.0 \%(0 / 8)$ of patients in the N-IRI and IRI groups, respectively.

\section{Discussion}

The primary endpoint of prolonged PFS was not achieved in this study, suggesting no significant benefit of N-IRI in non-biologically selected patients with AGC. This result is suggested by recent studies that evaluated the efficacy of anti-EGFR antibody administration to AGC patients who were not biologically selected. In two prospective randomized phase III studies (EXPAND, REAL-3) of cetuximab and panitumumab conducted in AGC patients, the primary endpoint could not be achieved [26, 27]. These negative results emphasize the need to identify the biological target before starting a large phase III study.

In a preclinical study, nimotuzumab showed marked antiproliferative, proapoptotic, and antiangiogenic effects against tumors showing EGFR overexpression [14-16]. We previously demonstrated that the effects of nimotuzumab on human NSCLC cell lines were highly dependent on EGFR status [28]. Nimotuzumab inhibited EGFR phosphorylation in cancer cells with high/moderate surface expression of EGFR, but not in those with low surface EGFR expression. Immunoblot analysis showed inhibition of EGFR phosphorylation in $\mathrm{H} 292$ and Ma-1 cells expressing high and moderate levels of EGFR on the cell surface, but not in H460, H1299, and H1975 cells showing a low level of surface EGFR expression [28].

In a clinical study of head and neck cancer to assess the efficacy of nimotuzumab in combination with radiotherapy, a controlled, double-blind, randomized clinical trial was conducted. For EGFR-positive patients, a significant survival improvement was detected for nimotuzumab-treated patients (OS, 16.5 months) compared with the control group (OS, 7.2 months) [29].

Nimotuzumab is a humanized IgG1 antibody-directed agent, meaning that EGFR should be considered as the first candidate for its biological target. In this study, subset analysis showed a median PFS of 118.5 days in the EGFR $2+/ 3+$ subgroup of the N-IRI group and 59.0 days in the corresponding subgroup of the IRI group; the RR was $33.3 \%$ and $0.0 \%$, respectively. Furthermore, there was no significant difference in the frequency and seriousness of adverse events between the IRI and N-IRI groups in the subset of EGFR 2+/3+ subgroup analysis. Submission of tissue samples was not mandatory, and EGFR protein expression was only detected for $57.3 \%$ of the full analysis set population. Therefore, the subset analysis based on the EGFR status could not yield any conclusive results. However, the results seem to imply that nimotuzumab can improve PFS and OS in AGC patients with high EGFR expression levels $(2+/ 3+)$ when administered in combination with irinotecan.

In our study, the further exploratory biomarker of $\mathrm{K}$-ras mutations was measured in 48 patients, and only 2 patients 
were found to harbor $K$-ras mutations. The EGFR gene copy number was measured in 46 patients, and 1 patient was detected with gene amplification. These results were consistent with previous reports $[30,31]$. The roles of $K$ ras mutations and $E G F R$ gene amplification were not clear in this study.

Recently, the ToGA study showed that the HER-2-targeting monoclonal antibody trastuzumab improved OS in AGC patients with HER-2 protein overexpression by IHC or gene amplification by FISH [10]. We also investigated the HER-2 expression levels by IHC and found that $14.6 \%$ (7/ 48) of patients showed HER-2 $2+13+$ expression and $29.2 \%$ (14/48) of patients showed EGFR $2+/ 3+$ expression. Only $2.1 \%(1 / 48)$ of patients showed $2+/ 3+$ expression of both EGFR and HER-2, suggesting there is little overlap between EGFR and HER-2 overexpression in gastric cancer [13]. Currently, targeted therapy for gastric cancer is limited to patients with HER-2 overexpression. However, in future, patients with gastric cancer showing EGFR overexpression might benefit from treatment with nimotuzumab.

In the present study, rash occurred in ten patients $(25.0 \%)$ in the N-IRI group, which represents a lower frequency than that reported for patients receiving other anti-EGFR monoclonal antibodies, such as cetuximab or panitumumab [26, 27]. Furthermore, there were no cases of severe ( $\geq$ grade 3 ) skin toxicity in either treatment group. The frequency and severity of skin toxicity associated with nimotuzumab appears to be lower than that associated with other anti-EGFR antibodies. The safety profile of nimotuzumab could be expected to maintain good quality of life as well as compliance and shows potential for combination of nimotuzumab with irinotecan. The median relative dose intensity of irinotecan and nimotuzumab was $94.94 \%$ and $96.55 \%$, respectively, in the N-IRI group. In the study of REAL-3, compliance with the baseline chemotherapy was decreased because of some severe toxicities, and the combination of a triple-chemotherapy regimen with panitumumab appears to be difficult to deliver [27].

The mechanism underlying this lower frequency of skin toxicity of nimotuzumab compared with that of other known anti-EGFR antibodies has been investigated in several recent studies [14-17, 19, 20]. These studies suggested that a low incidence of skin toxicity may be associated with the following: (1) the intermediate affinity $\left(K_{\mathrm{d}}=10^{-8} \mathrm{M}\right)$ of nimotuzumab, which is at least one order of magnitude lower than that of cetuximab or panitumumab; and (2) the different binding profile of nimotuzumab, which requires bivalent binding for stable attachment to the cellular surface compared with that of cetuximab, which requires only monovalent binding $[15,16]$. This finding implies that nimotuzumab binding to EGFR occurs only when the surface EGFR density is sufficiently high to allow bivalent binding. Tumor cells overexpressing EGFR are common, allowing for selective binding of nimotuzumab.

In conclusion, although the primary endpoint of prolonged PFS was not met in our study, subset analysis showed that the N-IRI regimen may have potential to improve PFS and OS in EGFR $2+/ 3+$ patients. An openlabel, randomized phase III trial comparing N-IRI and IRI in EGFR $2+/ 3+$ AGC patients is currently ongoing.

Acknowledgments We thank all the patients, their families, and the institutions involved in this study. The authors also thank the following individual board members for their contributions to this report: Independent Data Monitoring Committee, Keiichi Nagao, Noriyuki Masuda, Ichinosuke Hyodo, Baek-Yeol Ryoo, Young Iee Park, and Hong Suk Song; Efficacy Evaluation Committee, Atsushi Sato, Junji Tanaka, and Dae Young Zang; and Medical Advisors, Yutaka Ariyoshi and Kiyohiko Hatake. This trial was supported by a grant from Daiichi Sankyo Co., Ltd., Japan, and Kuhnil Pharm. Co., Ltd., Republic of Korea.

Conflict of interest T. Satoh, Y. Komatsu, Y. Yamada, and M. Munakata received research funding and honoraria from Daiichi Sankyo. Y.H. Kim received research funding and an honorarium from Kuhnil Pharm. K. Yamaguchi, N. Fuse, T. Ura, and S. Saitoh received research funding from Daiichi Sankyo. H. Yasui and S. Morita received honoraria from Daiichi Sankyo. Y. Sasaki, K. Nishio, and Y. Sakata received research funding, honoraria, and expert testimony from Daiichi Sankyo. K.H. Lee, S.Y. Rha, S.H. Park, T.Y. Kim, and S.Y. Kim have no conflicts of interests to declare.

Open Access This article is distributed under the terms of the Creative Commons Attribution License which permits any use, distribution, and reproduction in any medium, provided the original author(s) and the source are credited.

\section{References}

1. Murad AM, Santiago FF, Petroianu A, Rocha PR, Rodrigues MA, Rausch M. Modified therapy with 5-fluorouracil, doxorubicin, and methotrexate in advanced gastric cancer. Cancer (Phila). 1993;72:37-41.

2. Glimelius B, Ekström K, Hoffman K, Graf W, Sjödén PO, Haglund U, et al. Randomized comparison between chemotherapy plus best supportive care with best supportive care in advanced gastric cancer. Ann Oncol. 1997;8:163-8.

3. Wagner AD, Grothe W, Haerting J, Kleber G, Grothey A, Fleig WE. Chemotherapy in advanced gastric cancer: a systematic review and meta-analysis based on aggregate data. J Clin Oncol. 2006;24:2903-9.

4. Cunningham D, Starling N, Rao S, Iveson T, Nicolson M, Coxon $\mathrm{F}$, et al. Capecitabine and oxaliplatin for advanced esophagogastric cancer. N Engl J Med. 2008;358:36-46.

5. Kang YK, Kang WK, Shin DB, Chen J, Xiong J, Wang J, et al. Capecitabine/cisplatin versus 5-fluorouracil/cisplatin as first-line therapy in patients with advanced gastric cancer: a randomised phase III noninferiority trial. Ann Oncol. 2009;20:666-73.

6. Koizumi W, Narahara H, Hara T, Takagane A, Akiya T, Takagi $\mathrm{M}$, et al. S-1 plus cisplatin versus S-1 alone for first-line treatment of advanced gastric cancer (SPIRITS trial): a phase III trial. Lancet Oncol. 2008;9:215-21. 
7. Van Cutsem E, Moiseyenko VM, Tjulandin S, Majlis A, Constenla M, Boni C, et al. Phase III study of docetaxel and cisplatin plus fluorouracil compared with cisplatin and fluorouracil as firstline therapy for advanced gastric cancer: a report of the V325 Study Group. J Clin Oncol. 2006;24:4991-7.

8. Thuss-Patience PC, Kretzschmar A, Bichev D, Deist T, Hinke A, Breithaupt K, et al. Survival advantage for irinotecan versus best supportive care as second-line chemotherapy in gastric cancer: a randomised phase III study of the Arbeitsgemeinschaft Internistische Onkologie (AIO). Eur J Cancer. 2011;47:2306-14.

9. Kang JH, Lee SI, Lim DH, Park KW, Oh SY, Kwon HC, et al. Salvage chemotherapy for pretreated gastric cancer: a randomized phase III trial comparing chemotherapy plus best supportive care with best supportive care alone. J Clin Oncol. 2012;30:1513-8.

10. Bang YJ, Van Cutsem E, Feyereislova A, Chung HC, Shen L, Sawaki A, et al. Trastuzumab in combination with chemotherapy versus chemotherapy alone for treatment of HER2-positive advanced gastric or gastro-oesophageal junction cancer (ToGA): a phase 3, open-label, randomised controlled trial. Lancet. 2010;376:687-97.

11. Salomon DS, Brandt R, Ciardiello F, Normanno N. Epidermal growth factor-related peptides and their receptors in human malignancies. Crit Rev Oncol Hematol. 1995;19:183-232.

12. Kim MA, Lee HS, Lee HE, Jeon YK, Yang HK, Kim WH. EGFR in gastric carcinomas: prognostic significance of protein overexpression and high gene copy number. Histopathology (Oxf). 2008;52:738-46.

13. Terashima M, Kitada K, Ochiai A, Ichikawa W, Kurahashi I, Sakuramoto $\mathrm{S}$, et al. Impact of human epidermal growth factor receptor EGFR and ERBB2 on survival in stage II/III gastric cancer. Clin Cancer Res. 2012;18(21):5992-6000.

14. Crombet-Ramos T, Rak J, Pérez R, Viloria-Petit A. Antiproliferative, antiangiogenic and proapoptotic activity of h-R3: a humanized anti-EGFR antibody. Int J Cancer. 2001;101:567-75.

15. Talavera A, Friemann R, Gómez-Puerta S, Martinez-Fleites C, Garrido G, Rabasa A, et al. Nimotuzumab, an antitumor antibody that targets the epidermal growth factor receptor, blocks ligand binding while permitting the active receptor conformation. Cancer Res. 2009;69:5851-9.

16. Garrido G, Tikhomirov IA, Rabasa A, Yang E, Gracia E, Iznaga $\mathrm{N}$, et al. Bivalent binding by intermediate affinity of nimotuzumab: a contribution to explain antibody clinical profile. Cancer Biol Ther. 2011;11:373-82.

17. Crombet T, Torres L, Neninger E, Catalá M, Solano ME, Perera A, et al. Pharmacological evaluation of humanized anti-epidermal growth factor receptor, monoclonal antibody h-R3, in patients with advanced epithelial-derived cancer. J Immunother. 2003;26: 139-48.

18. Okamoto W, Yoshino T, Takahashi T, Okamoto I, Ueda S, Tsuya A, et al. A phase I, pharmacokinetic and pharmacodynamic study of nimotuzumab in Japanese patients with advanced solid tumors. Cancer Chemother Pharmacol. 2013;72:1063-71.

19. Crombet T, Osorio M, Cruz T, Roca C, del Castillo R, Mon R, et al. Use of the humanized anti-epidermal growth factor receptor monoclonal antibody h-R3 in combination with radiotherapy in the treatment of locally advanced head and neck cancer patients. J Clin Oncol. 2004;22:1646-54.

20. Ramos TC, Figueredo J, Catala M, González S, Selva JC, Cruz $\mathrm{TM}$, et al. Treatment of high-grade glioma patients with the humanized anti-epidermal growth factor receptor (EGFR) antibody h-R3: report from a phase I/II trial. Cancer Biol Ther. 2006;5:375-9.

21. Goldstein NS, Armin M. Epidermal growth factor receptor immunohistochemical reactivity in patients with American Joint Committee on Cancer Stage IV colon adenocarcinoma: implications for a standardized scoring system. Cancer (Phila). 2001;92: 1331-46.

22. Cappuzzo F, Hirsch FR, Rossi E, Bartolini S, Ceresoli GL, Bemis $\mathrm{L}$, et al. Epidermal growth factor receptor gene and protein and gefitinib sensitivity in non-small-cell lung cancer. J Natl Cancer Inst. 2005;97:643-55.

23. Chun JH, Kim HK, Lee JS, Choi JY, Lee HG, Yoon SM, et al. Weekly irinotecan in patients with metastatic gastric cancer failing cisplatin-based chemotherapy. Jpn J Clin Oncol. 2004;34:8-13.

24. Hironaka S, Zenda S, Boku N, Fukutomi A, Yoshino T, Onozawa Y. Weekly paclitaxel as second-line chemotherapy for advanced or recurrent gastric cancer. Gastric Cancer. 2006;9:14-8.

25. Kodera $Y$, Ito S, Mochizuki Y, Fujitake S, Koshikawa K, Kanyama Y, et al. A phase II study of weekly paclitaxel as second-line chemotherapy for advanced gastric cancer (CCOG0302 study). Anticancer Res. 2007;27:2667-71.

26. Lordick F, Kang YK, Chung HC, Salman P, Oh SC, Bodoky G, et al. Capecitabine and cisplatin with or without cetuximab for patients with previously untreated advanced gastric cancer (EXPAND): a randomised, open-label phase 3 trial. Lancet Oncol. 2013;14(6):490-9.

27. Waddell TS, Chau I, Cunningham D, Gonzalez D, Okines AF, Okines C, et al. Epirubicin, oxaliplatin, and capecitabine with or without panitumumab for patients with previously untreated advanced oesophagogastric cancer (REAL3): a randomised, open-label phase 3 trial. Lancet Oncol. 2013;14(6):481-9.

28. Akashi Y, Okamoto I, Iwasa T, Yoshida T, Suzuki M, Hatashita E, et al. Enhancement of the antitumor activity of ionising radiation by nimotuzumab, a humanised monoclonal antibody to the epidermal growth factor receptor, in non-small cell lung cancer cell lines of differing epidermal growth factor receptor status. $\mathrm{Br}$ J Cancer. 2008;98:749-55.

29. Rodríguez MO, Rivero TC, del Castillo Bahi R, Muchuli CR, Bilbao MA, Vinageras EN, et al. Nimotuzumab plus radiotherapy for unresectable squamous-cell carcinoma of the head and neck. Cancer Biol Ther. 2010;9(5):343-9.

30. Lee SH, Lee JW, Soung YH, Kim HS, Park WS, Kim SY, et al. BRAF and KRAS mutations in stomach cancer. Oncogene. 2003;22:6942-5.

31. Kim MA, Lee HS, Lee HE, Jeon YK, Yang HK, Kim WH. EGFR in gastric carcinomas: prognostic significance of protein overexpression and high gene copy number. Histopathology (Oxf). 2008;52(6):738-46. 